\title{
Foundation doctors' perspectives on the impact of the COVID-19 pandemic and lessons for the future
}

\author{
Authors: Prasanti A Kotta, ${ }^{\mathrm{A}}$ Madhivanan Elango, ${ }^{\mathrm{B}}$ Nitya Matcha ${ }^{\mathrm{A}}$ and Katarina YK Chow $^{\mathrm{A}}$
}

\begin{abstract}
The foundation programme is a 2-year training programme for newly qualified doctors and aims to bridge the gap between medical school and specialty training. The pandemic led to some major disruptions to foundation training. As foundation trainees, we encountered new challenges: there were reduced learning opportunities and our future paths became uncertain with changes to specialty training applications and membership exams. However, it is said that every crisis creates new opportunities and is a test of our resilience and innovativeness. There was the adoption of novel teaching methods, new research opportunities, increased importance given to teamwork and support for our wellbeing and mental health. We learnt lessons from this crisis that we should take forward to improve foundation training for the future.
\end{abstract}

KEYWORDS: training, education, wellbeing, foundation training, career planning

DOI: $10.7861 /$ clinmed.2021-0244

\section{Introduction}

The COVID-19 pandemic dramatically changed healthcare provision within the NHS, and the foundation training programme was not spared from the disruption. In the UK, the foundation programme is a 2-year training programme for newly qualified doctors. The programme aims to bridge the gap between medical school and specialty training, allowing newly qualified doctors to develop their clinical and professional skills.

The pandemic led to some major changes in foundation training. In April 2020, final-year medical students, a cohort that the authors were a part of, graduated early, received early provisional General Medical Council (GMC) registration and were given the opportunity to support the patient-facing staff. ${ }^{1}$ The transition from medical school to foundation training is already a big jump, with many doctors feeling anxious and nervous about

Authors: Afoundation year-1 doctor, St Thomas' Hospital, London, UK; ${ }^{B}$ foundation year-1 doctor, Imperial College London, London, UK

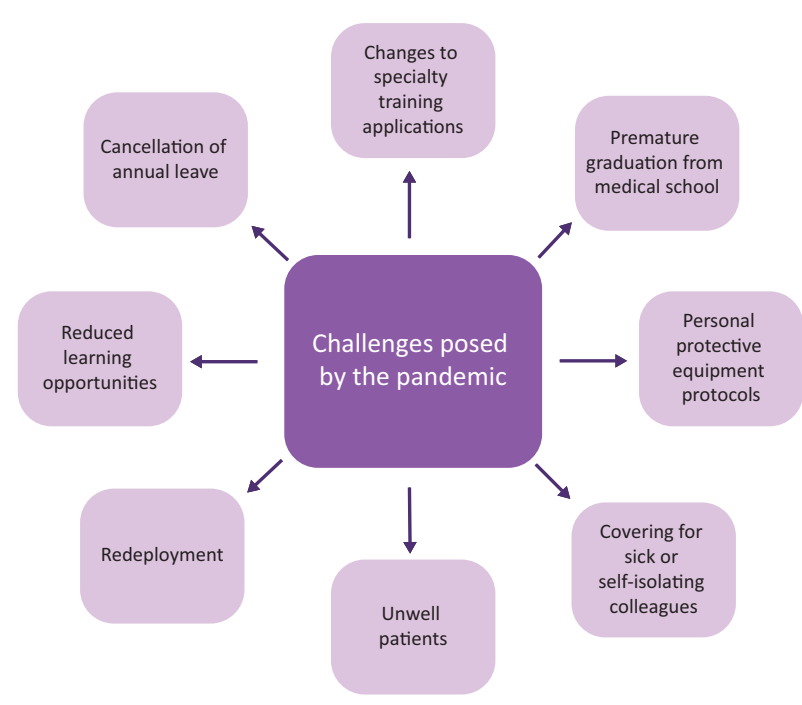

Fig 1. Challenges faced by foundation doctors during the COVID-19 pandemic.

it. $^{2}$ The pandemic posed several challenges to foundation doctors (Fig 1). ${ }^{3,4}$

Here, as foundation doctors, we reflect on the impact of the pandemic on our training, learning, wellbeing and career prospects. We discuss how the pandemic offers both challenges and opportunities, allowing us to adopt novel technologies and learn lessons that we can take forward to improve foundation training for the future.

\section{Impact on teaching}

Some mandatory teaching was initially cancelled as the majority of teaching was in person and not compatible with social distancing. The disruption to formal teaching was recognised by the annual review of competence progression (ARCP) panel with a reduction in the requirement of teaching attendance from 60 hours to 45 hours. $^{5}$

Foundation programmes soon began using novel video conferencing platforms such as Zoom and Microsoft Teams. Virtual teaching has advantages and disadvantages which are discussed in Fig 2. 

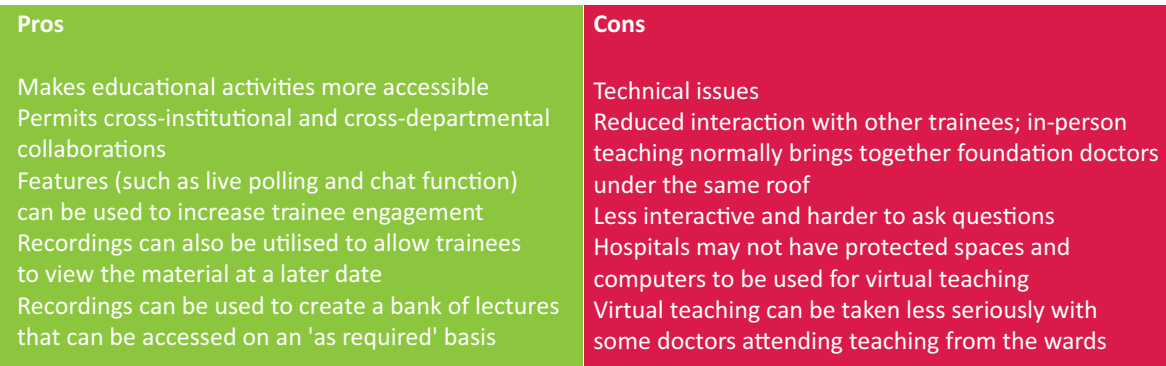

Fig 2. Pros and cons of virtual teaching.

We found that hospitals did not have protected spaces or electronic devices that we could use to attend teaching virtually. This meant that many of us attended virtual sessions from noisy ward environments using our mobile devices. Attending teaching sessions virtually from the wards took away from the 'protected' nature of dedicated learning time. Going forward, we believe that a careful balance needs to be struck with the provision of both in-person and virtual teaching to take advantage of both teaching methods.

\section{Impact on learning on the wards}

The pandemic offered ad hoc new learning opportunities such as critical care exposure, assessment and management of unwell patients, greater exposure to certain aspects of medical care (such as advance care planning (ACP)), and palliative and end-of-life care. COVID-19 was a novel disease with even the most senior clinicians feeling challenged. This led to a reduced sense of hierarchy and the whole team, from the consultants to foundation doctors, were learning and growing together. ${ }^{4}$ During ward rounds, foundation doctors were involved in checking local hospital and societal guidelines and informing other team members about the most up-to-date guidelines. The work by Coia and West emphasises the importance of autonomy, belonging and competence as key for wellbeing and motivation at work, and this change in the team dynamic helped enhance full engagement from foundation doctors. ${ }^{6}$ The 2020 barometer survey found that $62 \%$ of doctors experienced positive impacts on teamwork, $54 \%$ reported improved sharing of knowledge across the profession and $49 \%$ reported improved implementation of change. ${ }^{7}$

Foundation doctors who were redeployed to unfamiliar settings received education through simulation training, e-learning modules and webinars. ${ }^{8}$ We benefited from simulation training as it permitted us to learn at our own pace, in a stress-free environment, with immediate feedback. However, support for redeployed doctors could be improved by having an assigned clinical supervisor for redeployment, accessible senior support, creating support groups, daily consultant-led ward rounds and debrief sessions to discuss challenges encountered.

The pandemic also took away important learning opportunities. With hospitals being occupied by COVID-19 patients, there was a reduction in the variety of clinical cases seen. Some elective services were cancelled and many clinics became telephone or virtual clinics, resulting in reduced learning opportunities in certain specialties. ${ }^{4}$ Minimisation of patient interaction also resulted in reduced bedside teaching on clinical signs, examination skills and practical skills. Visitors of patients were also limited and we experienced less communication with both the patient and their families. ARCP requirements were adjusted to reflect this, the number of required case-based discussions (CBDs) reducing from six to four and the number of direct observations of doctor-patient interactions (DOPs) reducing from nine to six. ${ }^{5}$ We think this was a necessary change because patient interactions became mainly senior led, bedside teaching was minimised and redeployment meant that your team changed frequently making it harder to get sign-offs.

Advanced life support (ALS), normally a 2-day in-person course, was replaced by virtual learning modules and a single day practical course, and many foundation doctors found it difficult to ask questions. Teaching medical students is an important way for foundation doctors gain teaching skills and, with disruptions to medical student placements and emphasis on minimising patient contact, opportunities to teach medical students were also reduced.

\section{Impact on research}

The COVID-19 pandemic affected foundation doctor involvement in research. The pandemic necessitated the cancellation of many academic research blocks, redeployment of academic trainees to clinical service and suspension of some research not directly related to the COVID-19 pandemic. ${ }^{9}$ As a result, many academic trainees lost out on protected research time or had to change their research projects.

While some research opportunities were lost, new opportunities were created. The UK has been at the centre of large international collaborations on COVID-19 trials including the Randomised Evaluation of COVID-19 Therapy (RECOVERY) trial. Hospitals across the UK were involved in recruiting participants for these trials and foundation doctors benefited from exposure to research teams, trial recruitment and witnessed the rapid implementation of trial results to guide COVID-19 management. Some foundation doctors, after completing the necessary training, also became involved in the clinical trials, learning valuable new skills. ${ }^{10}$ This experience has inspired some of us to get involved in research. This model of offering research opportunities to foundation doctors and making research more accessible can help attract more foundation doctors to research.

The pandemic also impacted scientific conferences. Conferences allow foundation trainees to present their research while learning about other research and forming professional relationships. Due to the cancellation of many conferences, this networking opportunity was lost. However, many conferences became virtual, with some reducing or even eliminating their registration fees. ${ }^{11}$ Attending conferences can be prohibitively expensive for trainees due to the cost of registration fees, transportation 
and accommodation; free virtual academic conferences remove much of these barriers, potentially widening their outreach to trainees. Overall, it is clear that the initial shock of the pandemic forced much research activity to diminish. However, the pandemic also necessarily resulted in many research opportunities and, potentially, long-term changes that are likely to benefit junior doctors interested in research.

\section{Impact on career planning}

During the pandemic, many foundation doctors were redeployed to support the intensive care units or acute medical wards. In March 2020, Health Education England (HEE) made the announcement to cancel all foundation year rotations in April 2020 and foundation doctors remained in their current posts. ${ }^{12}$ This meant that foundation doctors lost the opportunity to gain experience in another specialty and this was especially devastating for those who missed rotating in a specialty of their choice, making portfolio development for specialty applications more difficult.

The pandemic also resulted in many professional exams being cancelled; postgraduate membership exams including the Membership of the Royal Colleges of Physicians (MRCP) and Membership of the Royal College of Surgeons (MRCS) were initially cancelled in the Spring of 2020 and were subsequently conducted online. ${ }^{13}$ This affected foundation doctors who had prepared for these exams and were hoping to utilise them to gain additional points for specialty recruitment.

Furthermore, there were significant changes to the specialty recruitment processes; interviews for certain training posts were shortened in length and conducted online, specialties (such as anaesthetics and paediatrics) newly introduced the Multi-Specialty Recruitment Assessment (MSRA) while others (such as general practice) have increased the weightage of the MSRA score as part of the recruitment process. These changes meant that those who had tailored their application towards the selection criteria of previous years were disappointed. ${ }^{14}$ The uncertainties caused by the evolving selection process, especially at a time when developing a portfolio and obtaining study leave became more difficult, contributed to anxiety among foundation trainees.

In recent years, statistics from the UK Foundation Programme suggest that there have been significant reductions in the number of doctors moving directly into specialty training after foundation training; in 2019 , only $34.9 \%$ of foundation doctors were appointed to specialty training, compared with 83\% back in 2010. Foundation doctors have instead been taking a year out, known colloquially as a foundation year 3 (F3), using it as an opportunity to gain more experience in certain specialties, travel and do locum work. We foresee the number of doctors taking an $\mathrm{F} 3$ rising as we come out of this pandemic as foundation doctors seek to recuperate from the stresses of working in a pandemic, especially in the cohort who experienced premature termination of medical school with minimal interleaving holiday. It will also be used to make up for missed clinical opportunities, prepare for professional exams and build portfolios for specialty applications.

\section{Impact on wellbeing}

Foundation doctors experienced significant and rapid changes to their personal and professional lives as a result of the pandemic including redeployment, changes to working patterns, isolating from family members and changes to medical school and

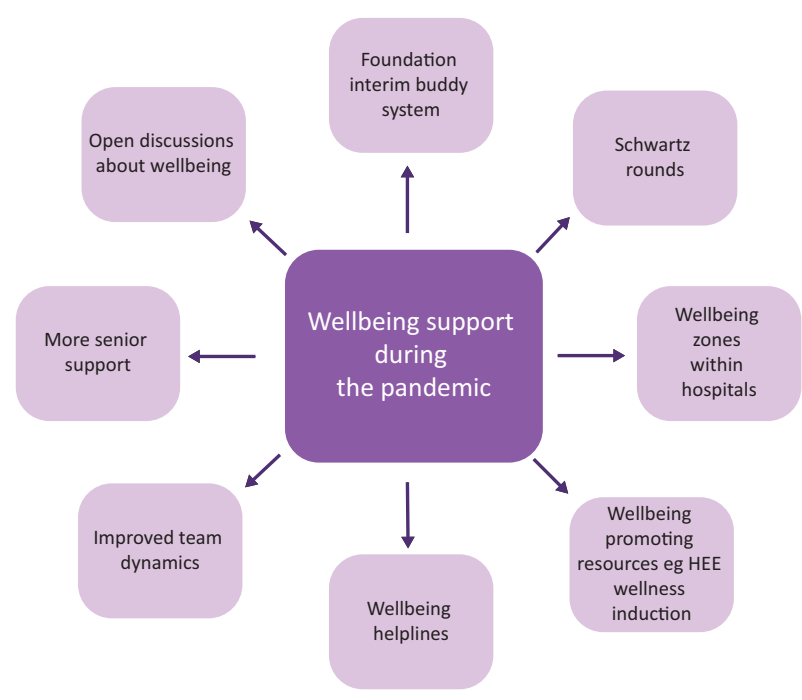

Fig 3. Strategies used to support foundation doctor wellbeing during the COVID-19 pandemic. HEE = Health Education England.

specialty training adjustments. The 2020 barometer survey found that $81 \%$ of doctors had significant changes to their working lives and $32 \%$ reported experiencing a negative impact on their mental health and wellbeing. ${ }^{7}$ In addition, around 7,000 final-year medical students were graduated early from medical school and were provisionally registered by the GMC to fill 4,662 newlycreated foundation interim year-1 (FiY1) posts between April and July 2020. Many of us missed our electives, celebrations of graduating from medical schools and the long-awaited holidays before starting work. Instead, nearly three-quarters (72\%) of FiY1 doctors worked in areas where there were confirmed or suspected cases of COVID-19. ${ }^{15}$

The crisis, despite the impact on safety and mental health, created new opportunities to support wellbeing. The 2020 barometer survey found that, compared with 2019, fewer doctors were struggling with high workloads (15\% compared with $26 \%$ ) and burnout (10\% compared with $16 \%$ ). ${ }^{7,16}$ The pandemic saw the rapid implementation of various wellbeing promoting strategies (Fig 3) and trainees felt supported with 38\% reporting more visibility of senior leaders. ${ }^{15}$ Going forward, these positive changes need to be sustained and embedded.

We also found that the foundation interim programme was very helpful, it gave us the opportunity to act as foundation year-1 doctors under close supervision. The buddying system gave us a well-supported start but the simultaneous treatment as a foundation doctor gave us the opportunity to learn quickly and acquire valuable skills. We think that the programme should serve as a model for designing the transition from medical school to foundation training.

\section{Conclusion}

The pandemic changed the way staff wellbeing is supported and training programmes are delivered. Adoption of novel methods (such as virtual teaching, simulation training, the involvement of foundation doctors in research and virtual academic conferences) and the importance of supporting staff wellbeing can all enhance the foundation training experience. However, it is important to 
recognise the unique needs of foundation doctors during the pandemic. We should seize the opportunity to be innovative, resilient and adaptive to find new ways to address the challenges that the pandemic has thrown at us and adapt to improve practices for the future.

\section{References}

1 UK Foundation Programme. 2020 recruitment stats and facts report. UK Foundation Programme, 2020.

2 Monrouxe LV, Grundy L, Mann M et al. How prepared are UK medical graduates for practice? A rapid review of the literature 2009-2014. BMJ Open 2017;7:e013656.

3 Sasitharan A. COVID-19: The impacts on foundation training in district general hospitals in the East of England and the East Midlands. Clin Med 2020:20:e253-4.

4 Salem J, Hawkins L, Sundaram A et al. COVID-19 and the Impact on Doctor Wellbeing and Training. Physician 2021;6:1-8.

5 UK Foundation Programme. Annual review of competence progression (ARCP) requirements guidance - all trainees. UK Foundation Programme, 2021

6 West M, Coia DD. Caring for doctors: Caring for patients: How to transform UK healthcare environments to support doctors and medical students to care for patients. General Medical Council, 2019.

7 Thompson J, Kearney I, Eastwood L, Cartmell B , Adams L. The State of Medical Education and Practice (SoMEP) Barometer survey 2020. General Medical Council, 2020.

8 Shelton C, Huda T, Lee A. The role of clinical simulation in preparing for a pandemic. BJA Educ 2021;21:172-9.

9 Ding A, Zhang Y. Impact of cancelling foundation year rotations due to the covid-19 outbreak in the UK. Postgrad Med J 2020;96:434
10 Carrington ZC. Why the RECOVERY trial should inspire foundation doctors to become involved in research - and for research teams to get them involved. BMJ 2020. Response to: Mahase E. Covid-19: Low dose steroid cuts death in ventilated patients by one third, trial finds. BMJ 2020;369:m2422.

11 Rich S, Diaconescu AO, Griffiths JD, Lankarany M. Ten simple rules for creating a brand-new virtual academic meeting (even amid a pandemic). PLoS Comput Biol 2020;16:e1008485.

12 Mangat P, Gardiner K, Parks R, MacLeod S, Melville C. Supporting the COVID-19 response: Plans for management of medical training rotations. GMC, 2020. www.gmc-uk.org/news/news-archive/ supporting-the-covid-19-response-plans-for-management-ofmedical-training-rotations

13 MacEwen C, Lawson T, MacLeod S et al. Exams for doctors in training - Academy of Medical Royal Colleges, COPMeD and GMC statement. GMC, 2020. www.gmc-uk.org/news/news-archive/ exams-for-doctors-in-training-a-joint-statement

14 Ooi SZY, Ooi R. Impact of the recent changes of the Multi-Specialty Recruitment Assessment (MSRA) weightage in specialty training recruitment during the COVID-19 pandemic. Postgrad Med J 2021; postgradmedj-2021-139808.

15 General Medical Council. The state of medical education and practice in the UK: 2020. GMC, 2020. www.gmc-uk.org/-/media/ documents/somep-2020_pdf-84684244.pdf

16 Thompson J, Kearney I, Stroud S, Adams L. The State of Medical Education and Practice (SoMEP) Barometer survey 2019. General Medical Council, 2019.

Address for correspondence: Dr Prasanti A Kotta, St Thomas' Hospital London, Westminster Bridge Road, South Bank, London SE1 7EH, UK.

Email: alekhya.kotta@kcl.ac.uk 